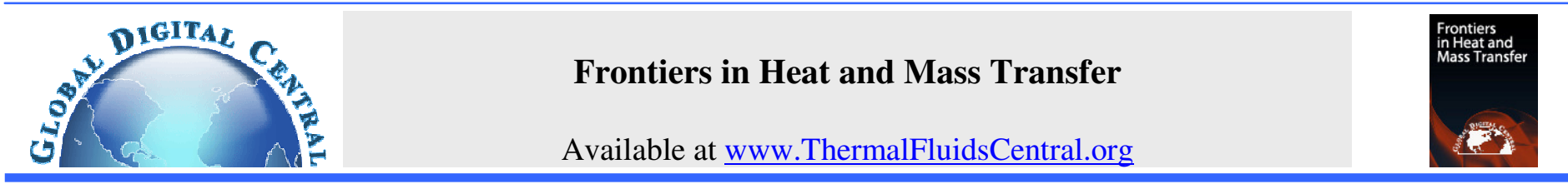

\title{
HEAT TRANSFER IN METAL FILMS IRRADIATED BY COMBINED NANOSECOND LASER PULSE AND FEMTOSECOND PULSE TRAIN
}

\author{
Yunpeng Ren, J. K. Chen", and Yuwen Zhang \\ Department of Mechanical and Aerospace Engineering, University of Missouri, Columbia, Missouri 65211, USA
}

\begin{abstract}
Heat transfer in a copper film irradiated by a femtosecond (fs) laser pulse train and by an integrated dual laser beam of a nanosecond pulse with a fspulse train was studied using the semi-classical two-temperature model. The critical point model with three Lorentzian terms was employed to characterize transient optical properties for the laser energy deposition. The effects of pulse number and separation time on the thermal response were investigated. The results showed that with the same total energy in a fs-pulse train, more pulses for shorter separation time, e.g., 1 ps, and fewer pulses for longer separation time, e.g., $100 \mathrm{ps}$, can achieve higher lattice temperature. For a dual laser beam, the lattice temperature can be increased by setting the pulse separation time as short as possible, e.g., 1 ps.
\end{abstract}

Keywords: semi-classical two-temperature model; femtosecond pulse train; dual laser beam, dynamic optical properties; critical point model.

\section{INTRODUCTION}

Femtosecond (fs) laser interactions with thin metal films have received intensive attention in the past two decades due to their unique characteristics in material processing (Baheti, 2010). To date, it has been possible to generate almost any arbitrary pulse shapes by recent development of optical devices. A pulse train generally consists of many 'trains' with a repetition rate of $\mathrm{KHz}$ to $\mathrm{MHz}$, in which each train may contain several pulses at a separation time of $100 \mathrm{fs}$ to hundreds of picoseconds (ps). In theoretical study, two-temperature models (TTM) (Anisimov et al., 1974; Chen and Beraun, 2001) are often employed to investigate the effects of pulse train on laser-material interactions (Huang et al., 2011; Jiang and Tsai, 2007; Sim et al., 2010; Jiang and Tsai, 2005; Li et al., 2009; Stoian et al., 2002; Chowdhury et al., 2003; Du et al., 2011).

Thermophysical and optical properties of materials are the two key factors that govern numerical solution accuracy in modeling ultrafast laser material interactions (Ren et al., 2012; Fisher et al., 2001; Ren et al., 2011). The former controls thermal transport and temperature distributions in a heated medium, while the latter dictates laser energy deposition that influences the thermal response. The dynamic change in surface reflectivity $(R)$ and absorption coefficient $(\alpha)$ due to temperature change caused by an ultrashort laser pulse could significantly alter the laser energy deposition for the succeeding pulses, leading to different thermal response than that described by using constant optical properties at room temperature. This may be particularly important for a solid target irradiated by a femtosecond pulse train or by an integrated dual laser beam of nanosecond (ns) and femtosecond pulses.

In this paper, a critical point (CP) model with three Lorentzian terms for interband transition (Etchegoin et al., 2006) is employed to describe transient optical properties of a metal film during laser pulse train irradiation. The semi-classical TTM (Chen et al., 2006) is proposed to simulate non-equilibrium heat transfer in the target. All the thermophysical properties employed are valid for a wide range of temperature (Ren et al., 2011). Numerical analyses are performed for a copper thin film. The effects of number of pulses in a single train and separation time between pulses on the thermal response are investigated. To understand the performance of dual ns-fs laser beams, the effects of change of the optical properties instigated by the addition of femtosecond laser pulses on the thermal response are also studied.

\section{SEMI-CLASSICAL TWO TEMPERATURE MODEL}

Consider that a free standing copper film of thickness $L$ and initial temperature $T_{i}$ is irradiated by a laser pulse train on the front surface ( $x$ $=0$ ). For simplicity, the problem is approximated to be one dimensional under the assumption that the laser spot size is much larger than the film thickness. Each femtosecond pulse train consists of several pulses with separation time of $t_{\text {sep }}$, and the repetition rate of trains is $f_{\text {rep }}$. The laser pulse is Gaussian in temporal with a FWHM (full width at half maximum) duration $t_{p}$.

Different from the TTM, the semi-classical TTM (Chen et al., 2006) considered the electron drifting effects. The three governing equations characterize the movement of electrons and the energy transport in the electrons and lattice. In the TTM modeling, the thermal conduction in lattice is often neglected for pure metals due to the fact that the thermal conductivity of lattice is much smaller than that of electrons. However, the heating process by a laser pulse train generally is much longer than that by a single pulse. Thus, the thermal conduction in the lattice could be important for precise description of the lattice response, especially when melting occurs. The semi-classical TTM considered in this work are given as follows:

$m \frac{\partial v}{\partial t}+m v \frac{\partial v}{\partial x}+\left[k_{B}\left(1+\frac{T_{e}}{C_{e}} \frac{\partial C_{e}}{\partial T_{e}}\right)-e \beta\right] \frac{\partial T_{e}}{\partial x}=-\frac{e T_{e} v}{\mu_{0} T_{l}}$

\footnotetext{
"Corresponding author. Email: ChenJnK@missouri.edu
} 
$C_{e}\left(\frac{\partial T_{e}}{\partial t}+v \frac{\partial T_{e}}{\partial x}+\frac{2}{3} T_{e} \frac{\partial v}{\partial x}\right)=\frac{\partial}{\partial x}\left(k_{e} \frac{\partial T_{e}}{\partial x}\right)-G\left(T_{e}-T_{l}\right)+S(x, t)$

$C_{l} \frac{\partial T_{l}}{\partial t}=\frac{\partial}{\partial x}\left(k_{l} \frac{\partial T_{l}}{\partial x}\right)+G\left(T_{e}-T_{l}\right)$

where $m$ is mass of an electron, $v$ electron drifting velocity, $t$ time, $k_{B}$ Boltzmann constant, $T$ temperature, $e$ charge of an electron, $C$ heat capacity, $\mu_{0}=4.8 \times 10^{-3}\left(\mathrm{~m}^{2} \mathrm{~s}^{-1} \mathrm{~V}^{-1}\right)$ mobility of electrons, $\beta=-1.42 \times 10^{-}$ ${ }^{4} T_{e} / T_{F}$ (V/K) electric field coefficient with Fermi temperature $T_{F}, k$ thermal conductivity, $G$ electron-phonon coupling factor, and $S$ laser heat density. The subscript $e$ and $l$ denote electron and lattice, respectively. The laser heat density in Eq. (2) for the entire pulse train can be expressed as:

$$
\begin{aligned}
& S(x, t)=\sum_{i=1}^{K} \sum_{j=1}^{N} 0.94 \frac{[1-R(0, t)] J_{i j}}{t_{p}} \alpha(x, t) \\
& \exp \left[-\int_{0}^{x} \alpha(x, t) d x-2.77\left(\frac{t-\frac{i-1}{f_{\text {rep }}}-(j-1) t_{\text {sep }}-2 t_{p}}{t_{p}}\right)^{2}\right]
\end{aligned}
$$

where $K$ is the number of trains, $N$ is the number of pulses in each train, $R(0, t)$ is surface reflectivity, $\alpha(x, t)$ is absorption coefficient, and $J$ is laser pulse fluence. Both the surface reflectivity and absorption coefficient could vary from pulse to pulse even though all the pulse fluences are identical $\left(J_{i j}=\right.$ constant $)$. The lasing time of each pulse is assumed to be $4 t_{p}$ because the laser power outside this time period is small.

\section{ULTRFAST SOLID-LIQUID PHASE CHANGE}

During a laser pulse train heating, melting and re-solidification could take place if pulse fluences are sufficiently high. The solid-liquid (S-L) phase change induced by ultrashort laser pulses is controlled by nucleation dynamics, instead of energy balance. The S-L interface can be heated well above the normal melting point during the melting process, in which case the solid becomes superheated. Similarly, the interface can be cooled far below the normal melting point in the solidification process, in which case the liquid becomes undercooled.

The energy balance at the S-L interface is in the form (Faghri and Zhang, 2006):

$k_{l, s} \frac{\partial T_{l, s}}{\partial x}-k_{l, \ell} \frac{\partial T_{l, \ell}}{\partial x}=\rho_{\ell} h_{m} u_{s \ell}$

where the subscripts $s$ and $\ell$ are associated with solid and liquid, respectively; $\rho$ is mass density; $h_{m}$ is latent heat of fusion; $u_{s \ell}$ is the $S$ -

$\mathrm{L}$ interfacial velocity. For a rapid melting/re-solidification process controlled by nucleation dynamics, the $\mathrm{S}-\mathrm{L}$ interfacial velocity can be computed by (Kuo and Qiu, 1996):

$u_{s \ell}=V_{0}\left[1-\exp \left(-\frac{h_{m}}{R_{g} T_{m}} \frac{T_{l, I}-T_{m}}{T_{l, I}}\right)\right]$

where $R_{g}$ is the gas constant for metals, $T_{m}$ is normal melting point, $T_{l, I}$ is interfacial temperature, and $V_{0}=u_{0} \exp \left(-h_{m} / R_{g} T_{m}\right)$ with $u_{o}$ being the maximum interfacial velocity. For copper, $V_{0}=1302.8 \mathrm{~m} / \mathrm{s}$.

\section{OPTICAL AND THERMOPHYSICAL PROPERTIES}

Recently, the CP model with three Lorentzian terms was validated experimentally by Ren et al. (2011) for copper, showing that the surface reflectivity and absorption coefficient can be accurately characterized for laser wavelength of $200-1000 \mathrm{~nm}$. It is thus adopted here to describe dielectric permittivity:

$$
\begin{aligned}
\varepsilon & =\varepsilon_{\infty}-\frac{\omega_{D}^{2}}{\omega^{2}+i \gamma \omega}+\sum_{p=1}^{3} B_{p} \Omega_{p}\left(\frac{e^{i \phi_{p}}}{\Omega_{p}-\omega-i \Gamma_{p}}+\frac{e^{-i \phi_{p}}}{\Omega_{p}+\omega+i \Gamma_{p}}\right) \\
& =\varepsilon_{1}(x, t)+i \varepsilon_{2}(x, t)
\end{aligned}
$$

where $\varepsilon_{\infty}$ is dielectric constant, $\omega_{D}$ plasma frequency, $\omega$ laser frequency, $\gamma$ damping coefficient which equals reciprocal of electron relaxation time $\left(\tau_{e}\right), B$ a weighting factor, and $\Omega, \phi$ and $\Gamma$ energy of gap, phase and broadening, respectively. The constants in Eq. (7) for copper can be found in Ren et al. (2011).

The optical properties $R$ and $\alpha$ can be determined from Fresnel function (Fox, 2010):

$$
R(x, t)=\frac{\left.\left(f_{1}-1\right)^{2}+f_{2}^{2}\right)}{\left(f_{1}+1\right)^{2}+f_{2}^{2}} ; \quad \alpha(x, t)=\frac{2 \omega f_{2}}{c}
$$

where $c$ is light speed in vacuum, and the normal refractive index $f_{l}$ and extinction coefficient $f_{2}$ are functions of $\varepsilon_{1}$ and $\varepsilon_{2}$ :

$f_{1}(x, t)=\sqrt{\frac{\varepsilon_{1}+\sqrt{\varepsilon_{1}^{2}+\varepsilon_{2}^{2}}}{2}} ; \quad f_{2}(x, t)=\sqrt{\frac{-\varepsilon_{1}+\sqrt{\varepsilon_{1}^{2}+\varepsilon_{2}^{2}}}{2}}$

The thermophysical properties such as electron heat capacity $\left(C_{e}\right)$, electron relaxation time $\left(\tau_{e}\right)$, and electron-phonon coupling factor $(G)$ employed in this work are valid for a wide range of temperature. Their analytical expressions are as follows (Ren et al., 2011):

$$
\begin{aligned}
& C_{e}\left(T_{e}\right)=\left\{\begin{array}{l}
117.47 T_{e}, \quad T_{e}<2 \times 10^{3} K \\
-2.049 \times 10^{4}-26.64 T_{e}+0.1 T_{e}^{2}-1.122 \times 10^{-5} T_{e}^{3}+ \\
5.735 \times 10^{-10} T_{e}^{4}-1.524 \times 10^{-14} T_{e}^{5}+2.044 \times 10^{-19} \\
T_{e}^{6}-1.094 \times 10^{-24} T_{e}^{7}, 2 \times 10^{3} K \leq T_{e} \leq 50 \times 10^{3} K
\end{array}\right. \\
& G\left(T_{e}\right)=\left\{\begin{array}{l}
0.56 \times 10^{17}, \quad T_{e}<2,750 K \\
1.341 \times 10^{17}-1.407 \times 10^{14} T_{e}+5.988 \times 10^{10} T_{e}^{2}-7.93 \\
\times 10^{6} T_{e}^{3}+555.2 T_{e}^{4}-0.0233 T_{e}^{5}+6.041 \times 10^{-7} T_{e}^{6}- \\
9.529 \times 10^{-12} T_{e}^{7}+8.377 \times 10^{-17} T_{e}-38.15 \times 10^{-22} \\
T_{e}^{9}, 2,750 K \leq T_{e} \leq 50 \times 10^{3} K
\end{array}\right. \\
& \tau_{e}=\frac{1}{3.54 \times v_{e, p h}+A_{e} T_{e}^{2}}
\end{aligned}
$$

with

$$
\begin{aligned}
v_{e, p h}= & \frac{\Xi^{2}}{8 \pi \varepsilon_{F} k_{F} \rho s} \frac{m_{o p t}}{m}\left\{\int_{0}^{q_{b}} \frac{e^{\phi_{l}}+e^{\phi_{e}}}{\left(e^{\phi_{l}}-1\right)\left(e^{\phi_{e}}+1\right)} q^{4} d q+\right. \\
& \eta \int_{0}^{q_{b}} \frac{e^{\phi_{l}}-e^{\phi_{e}}}{\left(e^{\phi_{l}}-1\right)\left(e^{\phi_{e}}+1\right)} q^{3} d q+q_{b} \frac{e^{\varphi_{l}}+e^{\varphi_{e}}}{\left(e^{\varphi_{l}}-1\right)\left(e^{\varphi_{e}}+1\right)} \times \\
& \left.\frac{\left(2 k_{F}\right)^{4}-q_{b}^{4}}{4}-4 \eta q_{b}^{2} k_{F}^{2} \frac{e^{\varphi_{l}}-e^{\varphi_{e}}}{\left(e^{\varphi_{l}}-1\right)\left(e^{\varphi_{e}}+1\right)}\right\}
\end{aligned}
$$

where $\phi_{l}=\beta_{l} \hbar q s, \phi_{e}=\beta_{e} \hbar q s, \varphi_{l}=\beta_{l} \hbar q_{b} s, \varphi_{e}=\beta_{e} \hbar q_{b} s, \varepsilon_{F}$ is Fermi energy, $k_{F}$ Fermi velocity, $\rho$ density, $s$ longitudinal sound velocity, $m_{\text {opt }}$ effective electron mass, $m$ mass of an electron, $q$ the phonon wave 
vector, $\beta_{l}=T_{l} / k_{B}, \beta_{e}=T_{e} / k_{B}$, and $\eta=2 m_{o p t} s / \hbar$. For copper, $\Xi=$ $3.99 \mathrm{eV}, q_{b}=8.97 \times 10^{9} \mathrm{~m}^{-1}$ with $m_{\text {opt }}=1.39 \mathrm{~m}$. The constant 3.54 is to cater to the relaxation time of $10.0 \mathrm{fs}$ at room temperature (Ren et al., 2011). The electron thermal conductivity $\left(k_{e}\right)$ is given by (Anisimov and Rethfeld, 1997):

$k_{e}=\chi \frac{\left(\vartheta_{e}^{2}+0.16\right)^{5 / 4}\left(\vartheta_{e}^{2}+0.44\right) \vartheta_{e}}{\left(\vartheta_{e}^{2}+0.092\right)^{1 / 2}\left(\vartheta_{e}^{2}+\zeta \vartheta_{l}\right)}$

where $\vartheta_{e}=T_{e} / T_{F}$ and $\vartheta_{l}=T_{l} / T_{F}$ with $T_{F}$ denoting Fermi temperature; $\chi$ and $\eta$ are constant. For copper, $T_{F}=8.16 \times 10^{4} \mathrm{~K}, \chi=$ $377 \mathrm{Wm}^{-1} \mathrm{~K}^{-1}$, and $\zeta=0.139$ (Chen and Beraun, 2005).

The bulk thermal conductivity $\left(k_{e q}\right)$ is the sum of electronic component $\left(k_{e}\right)$ and lattice component $\left(k_{l}\right)$. For pure metals, $k_{l}$ is much smaller than $k_{e}$ and is taken to be $1 \%$ of $k_{e q}$ for copper in this work (Klemens and Williams, 1986). The temperature-dependent bulk thermal conductivity, specific heat and mass density of copper in solid and liquid phase can be found in Ren et al. (2011).

\section{NUMERICAL ALGORITHM}

The electron momentum equation, electron energy equation, lattice energy equation, and the equations for phase change are solved numerically using a finite difference method. In each time step, the laser heat density is evaluated with the values of $R(0, t)$ and $\alpha(x, t)$ that are obtained from Eqs. (7)-(9) with electron and lattice temperature at the previous time step. Meanwhile, the dynamic thermophysical properties are updated. Equations (1)-(3) are then solved for new electron drifting velocity and temperatures of electrons and lattice with the updated laser heat density and thermophysical properties. If either melting or re-solidification occurs, the S-L interfacial velocity, temperature and location are calculated using the numerical method proposed by Zhang and Chen (2008). After that, the electron and lattice temperature fields are solved again from Eqs. (1)-(3). The new temperature fields are used to re-assess the phase change. The above procedure is repeated until the maximum difference of the temperatures between two successive iterations is less than $10^{-5} \mathrm{~K}$. Then, the calculation is marched to the next time step.

\section{RESULTS AND DISCUSSION}

The numerical analyses are performed for a copper film of $1-\mu \mathrm{m}$ thickness at an initial temperature of $300 \mathrm{~K}$. The effects of number and separation time of laser pulses are first studied for the film heated by a single laser train. Two different femtosecond laser trains are considered: one consists of three pulses and the other six pulses. All the pulses in each train have the same fluence, wavelength $800 \mathrm{~nm}$, and duration $100 \mathrm{fs}$. The total fluence is $1.0 \mathrm{~J} / \mathrm{cm}^{2}$ for all the trains, and three separation times between pulses are 1,10 and $100 \mathrm{ps}$. Then, the effects of combined nanosecond laser pulse and fs-pulse train are investigated. The parameters of the nanosecond laser pulse are: $\lambda=800$ $\mathrm{nm}, t_{p}=1 \mathrm{~ns}$, and fluence $0.5 \mathrm{~J} / \mathrm{cm}^{2}$.

Figure 1 shows the time evolution of electron and lattice temperature at the surface irradiated by the two femtosecond laser trains, respectively. The pulse separation time considered here is 100 ps. For comparison, the results for the film irradiated by a single femtosecond pulse of fluence $1.0 \mathrm{~J} / \mathrm{cm}^{2}$, same as the total fluence of each train, are also presented in Fig. 1(a). It can be seen in Figs. 1(b) and 1(c) that in each case the peak electron temperatures produced by the pulses are gradually increasing due to the incubation effect. However, the highest electron temperature decreases with increase of pulse number. This is mainly because of the lower laser energy carried by each pulse in the train with more pulses, leading to a smaller change of surface reflectivity as shown in Fig. 2. The maximum surface

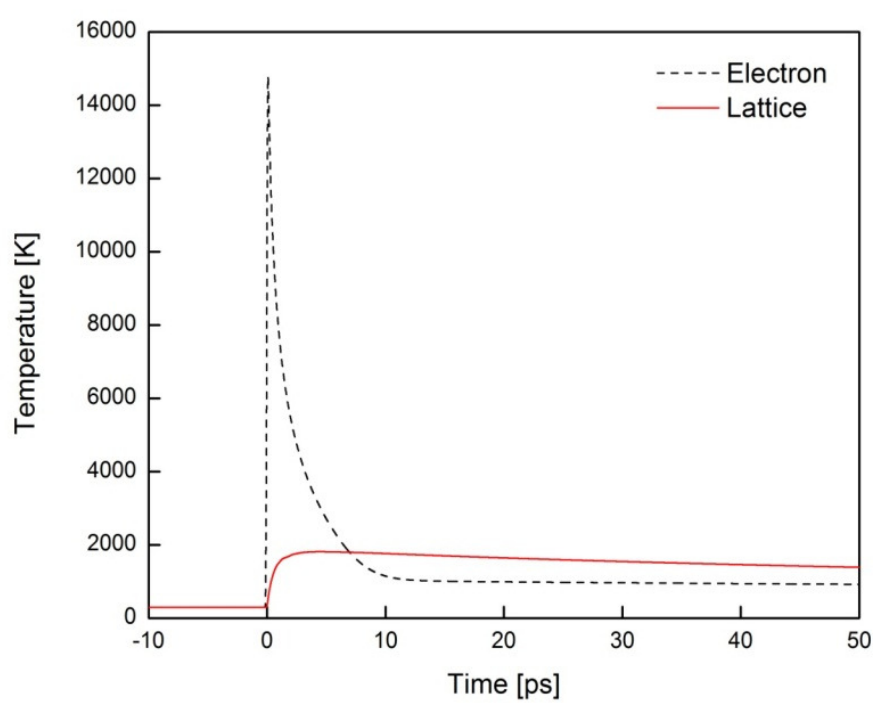

(a)

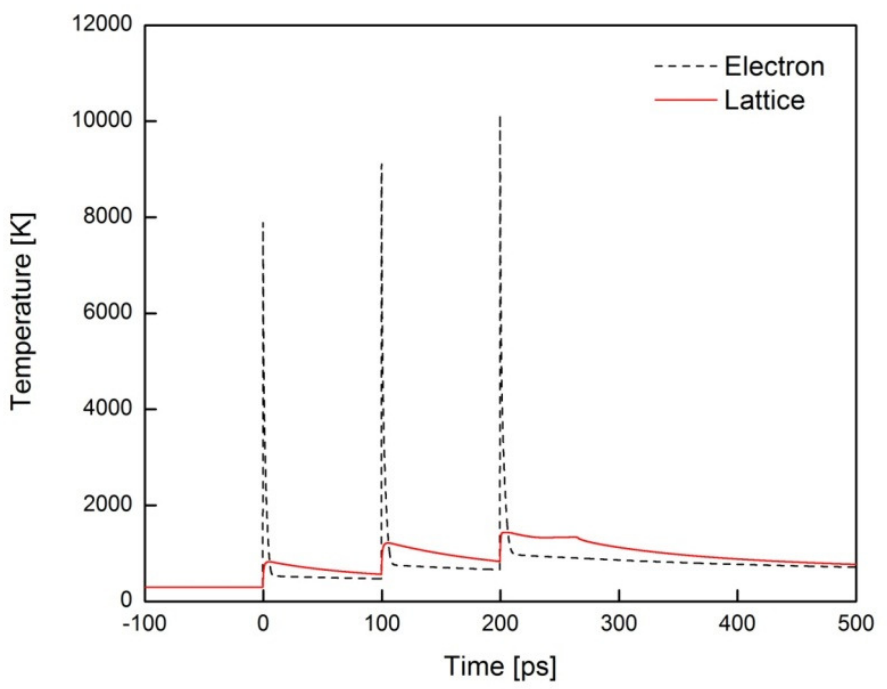

(b)

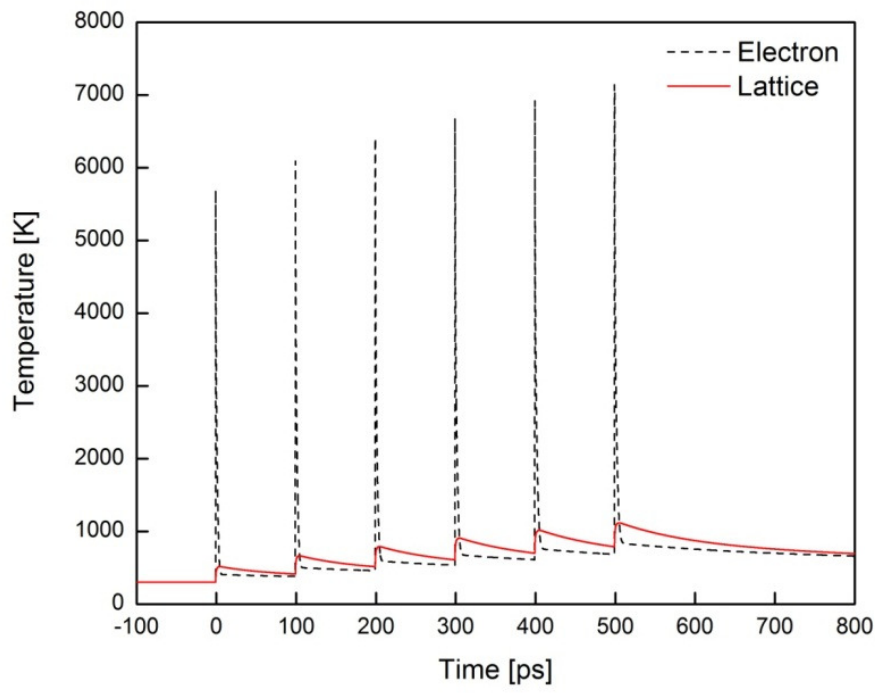

(c)

Fig. 1 Evolutions of electron and lattice temperature at the irradiated surface by femtosecond laser pulse trains with total fluence $J=$ $1.0 \mathrm{~J} / \mathrm{cm}^{2}$ and $t_{\text {sep }}=100 \mathrm{ps}$ : (a) single pulse, (b) three pulses, and (c) six pulses. 


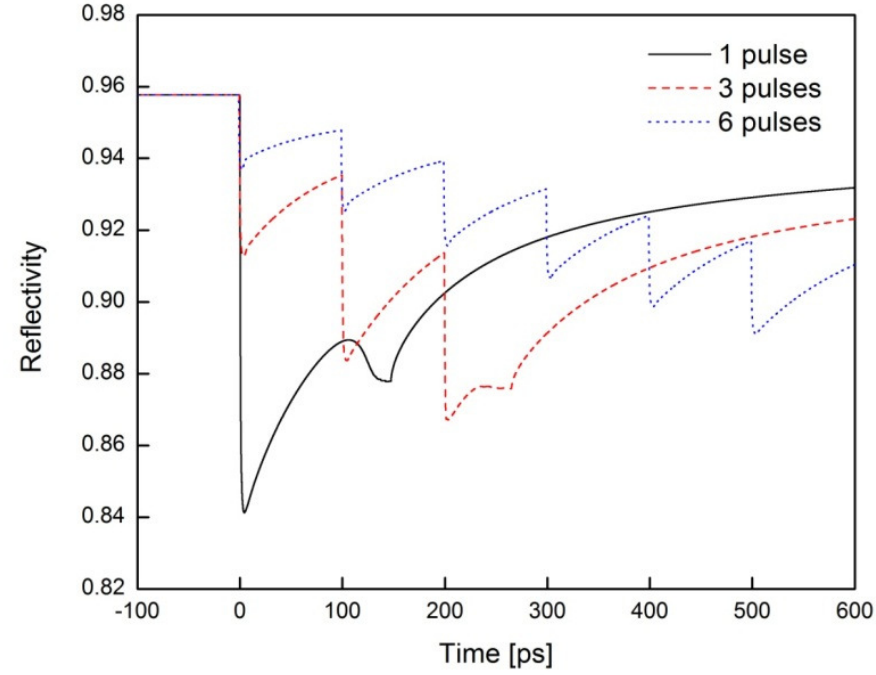

Fig. 2 Changes of surface reflectivity by different pulse trains with $t_{s e p}$ $=100 \mathrm{ps}$.

reflectivity is changed from 0.958 at room temperature to 0.841 for the single pulse, 0.867 for the three pulses train, and 0.891 for the six pulses train. The smaller change in surface reflectivity results in less laser energy deposition into the film, and thereby generating lower electron temperature. The highest lattice temperatures for the three cases are $1814 \mathrm{~K}, 1438 \mathrm{~K}$ and $1118 \mathrm{~K}$, respectively. The small bump in the lattice temperature at about $t=260 \mathrm{ps}$ in Fig. 1(b) is due to resolidification. In addition to the less laser energy absorption, the lower lattice temperature produced by a train with more pulses results from slower thermal energy transferred from electrons to the lattice due to the smaller electron-phonon coupling. This suggests that for a relatively long pulse separation time, for example $100 \mathrm{ps}$, a train with fewer pulses could generate higher lattice temperature should the total laser fluences in each train be identical. It is also found that the changes in absorption coefficient simulated are much less significant, as compared to those in surface reflectivity.

Figure 3 demonstrates the influence of pulse separation time on the electron and lattice temperature for $t_{\text {sep }}=1 \mathrm{ps}$ and $10 \mathrm{ps}$. Comparing the results in Figs. 1 and 3 shows that the highest electron temperature increases with decrease of separation time, and so does the lattice temperature. It, however, is interesting to note the maximum lattice temperatures: $2441 \mathrm{~K}$ for the three pulses and $2532 \mathrm{~K}$ for the six pulses in the 1-ps separation time case and $2282 \mathrm{~K}$ and $1961 \mathrm{~K}$ in the 10-ps separation time case. The above results along with those in the 100-ps separation time case imply that heat conduction in electrons and lattice is another important factor that affects temperature and optical response. For a shorter pulse separation time, the electrons and lattice may not have enough time to transfer most of the thermal energy to deeper parts of material before the next pulse arriving. Thus, the incubation effect could be more significant for a laser train with more pulses. This can be seen from Fig. 4 that depicts the transient change of surface reflectivity. The orders of the maximum change in the surface reflectivity are: 6, 3 and 1 pulse for the 1-ps separation time (Fig. 4(a)), 3, 6 and 1 pulse for the 10-ps separation time (Fig. 4(b)), and 1, 3 and 6 pulses for the 100-ps separation time (Fig. 2). These results of surface reflectivity give further explanation for the thermal responses shown in Figs. 1 and 3. To obtain higher lattice temperature, more pulses should be used in a train for shorter separation time such as 1 ps and fewer pulses should be used for longer pulse separation time such as $100 \mathrm{ps}$.

Figure 5 compares the lattice temperatures at the irradiated surface by a combined nanosecond and fs-laser pulse and by the nanosecond pulse alone. It is found that the two maximum lattice temperatures are $489 \mathrm{~K}$ and $2153 \mathrm{~K}$. The rise of temperature by the nanosecond laser pulse alone is $189 \mathrm{~K}$. Recall that the peak lattice temperature induced by the femtosecond pulse alone is $1814 \mathrm{~K}$ (Fig. 1). It thus appears that an integrated fs-ns dual beam can enhance thermal response through optical property changes by adding a nanosecond pulse. The bump shown in Fig. 5 is due to material solidification.

The lattice temperatures at the irradiated surface by the dual beams of a nanosecond pulse with a three fs-pulses train are shown in Fig. 6 for three pulse separation times: 1 ps, 10 ps and 100 ps. As expected, the lattice temperature decreases as the pulse separation time increases. The highest temperatures are $2793 \mathrm{~K}, 2656 \mathrm{~K}, 1741 \mathrm{~K}$, respectively. The change of rise of the lattice temperature is increased by $16 \%$ for the 1-ps separation time, $19 \%$ for the 10-ps separation time, and $27 \%$ for the 100-ps separation time, respectively, as compared to those cases of the three-femtosecond pulse trains in Figs. 1(c) and 3. Recall that the peak lattice temperature is $2153 \mathrm{~K}$ for the combined nanosecond and femtosecond pulse (Fig. 5) and $1814 \mathrm{~K}$ for the femtosecond pulse alone (Fig. 1). These results indicates that for a dual beam with a 3 fs-pulses train, the shorter the separation time is, the more the lattice temperature increases

Figure 7 shows the lattice temperatures at the irradiated surface by a dual beam of the nanosecond pulse and a 6 fs-pulses train with separation time of $10 \mathrm{ps}$. In this case, the highest lattice temperature is

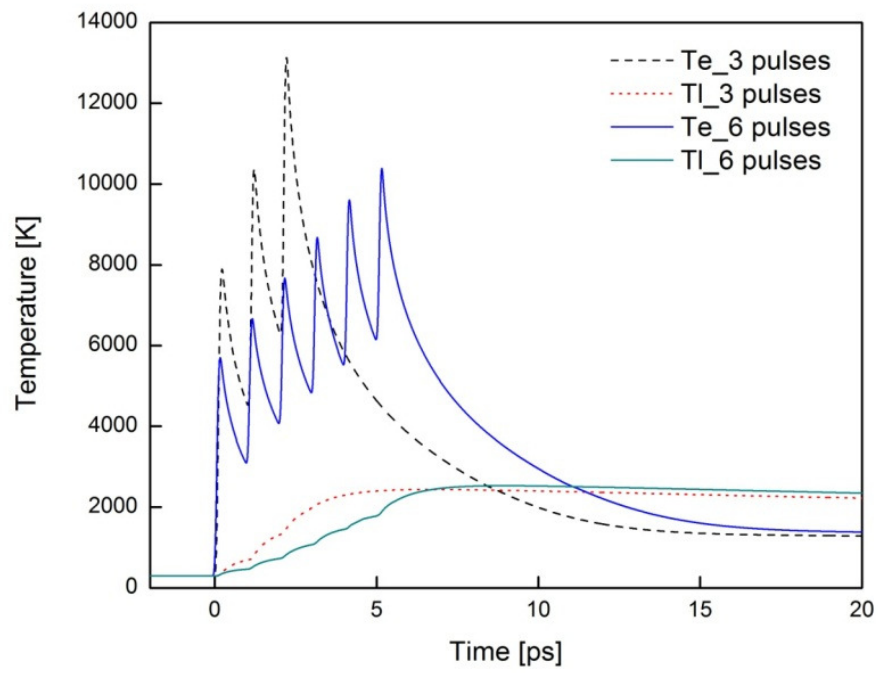

(a)

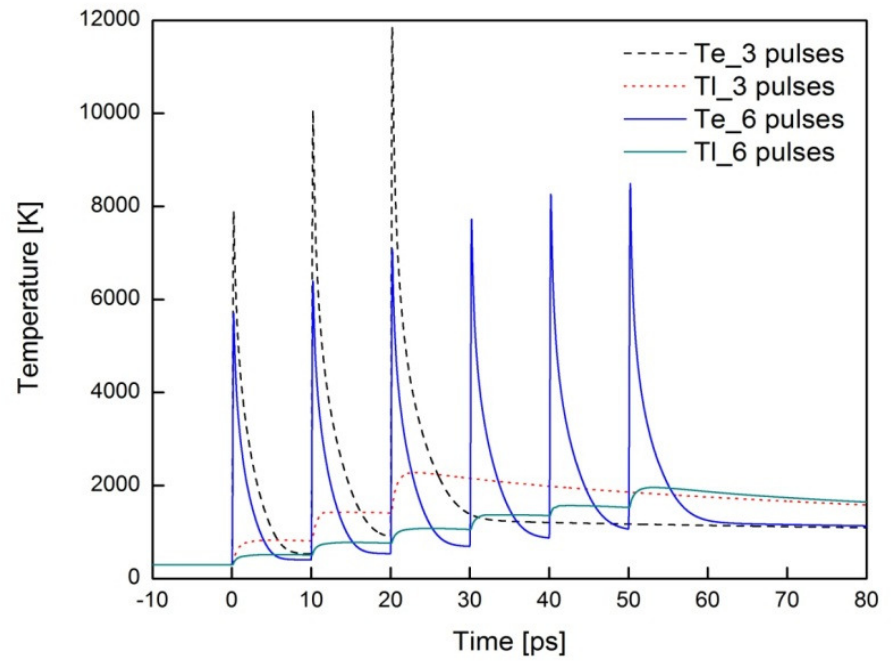

(b)

Fig. 3 Time histories of electron and lattice temperature at the irradiated surface by femtosecond laser pulse trains with total fluence $J=1.0 \mathrm{~J} / \mathrm{cm}^{2}$ : (a) $t_{\text {sep }}=1 \mathrm{ps}$ and (b) $t_{\text {sep }}=10 \mathrm{ps}$. 


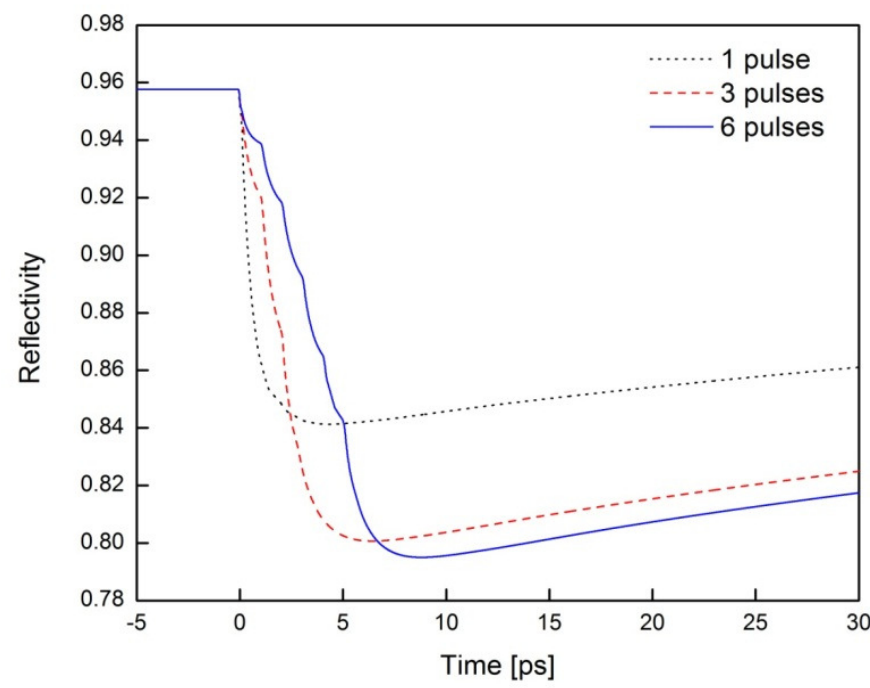

(a)

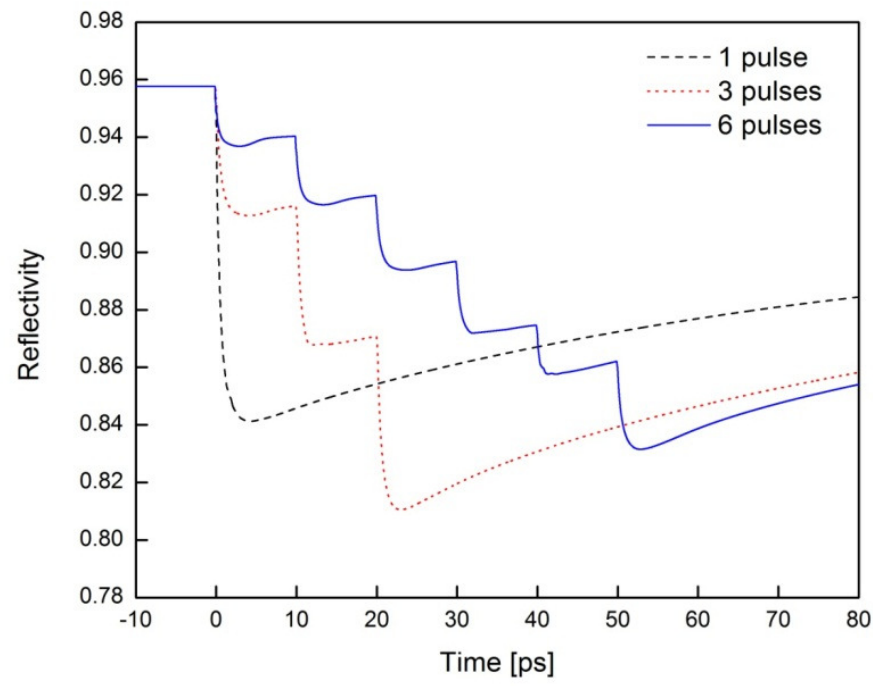

(b)

Fig. 4 Change of surface reflectivity by femtosecond laser pulse trains with total fluence $J=1.0 \mathrm{~J} / \mathrm{cm}^{2}$ : (a) $t_{\text {sep }}=1 \mathrm{ps}$ and (b) $t_{\text {sep }}=10$ ps.

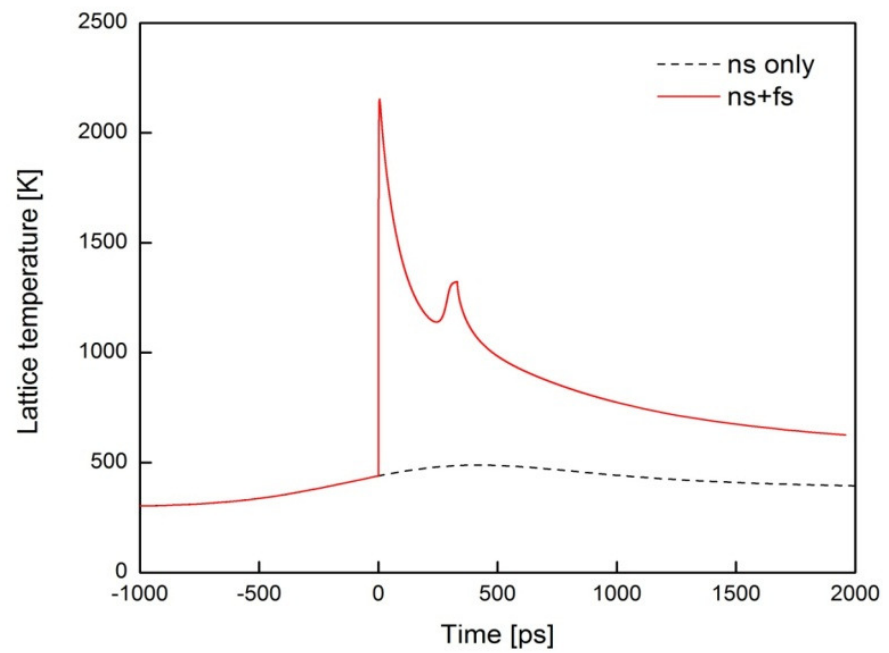

Fig. 5 Lattice temperature at the irradiated surface induced by a dual ns-fs laser beam and by the nanosecond pulse alone.

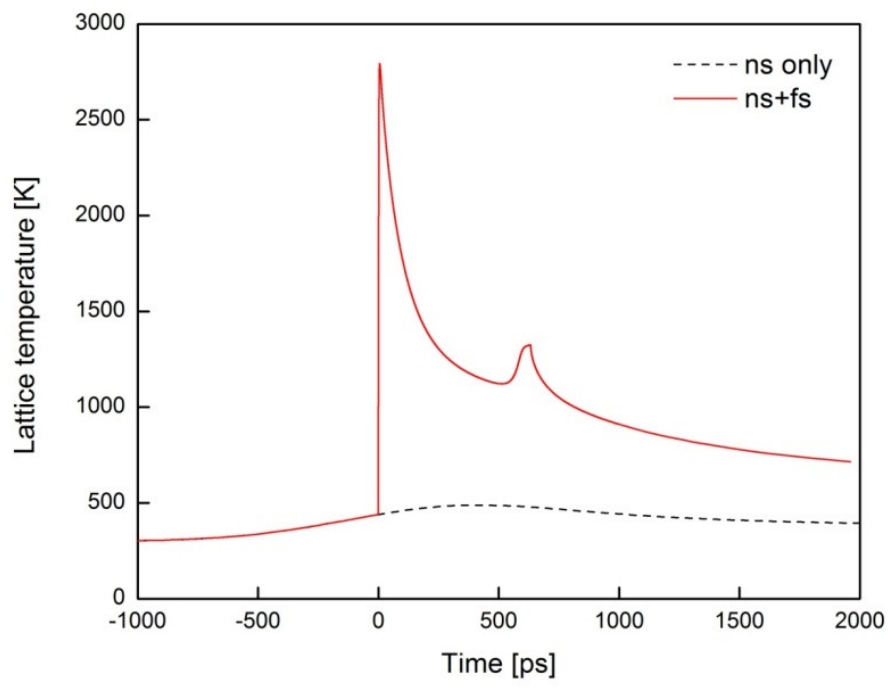

(a)

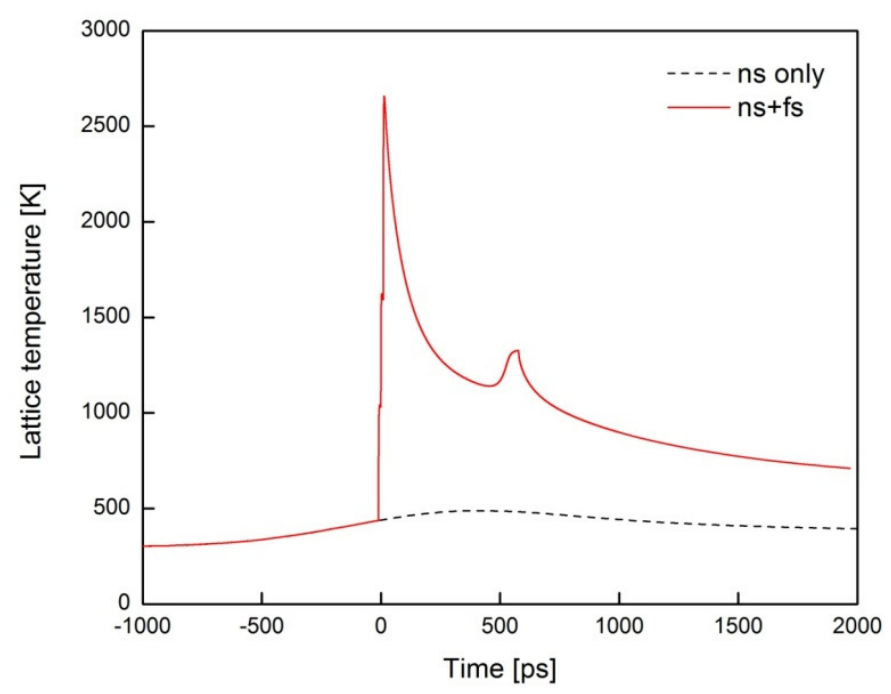

(b)

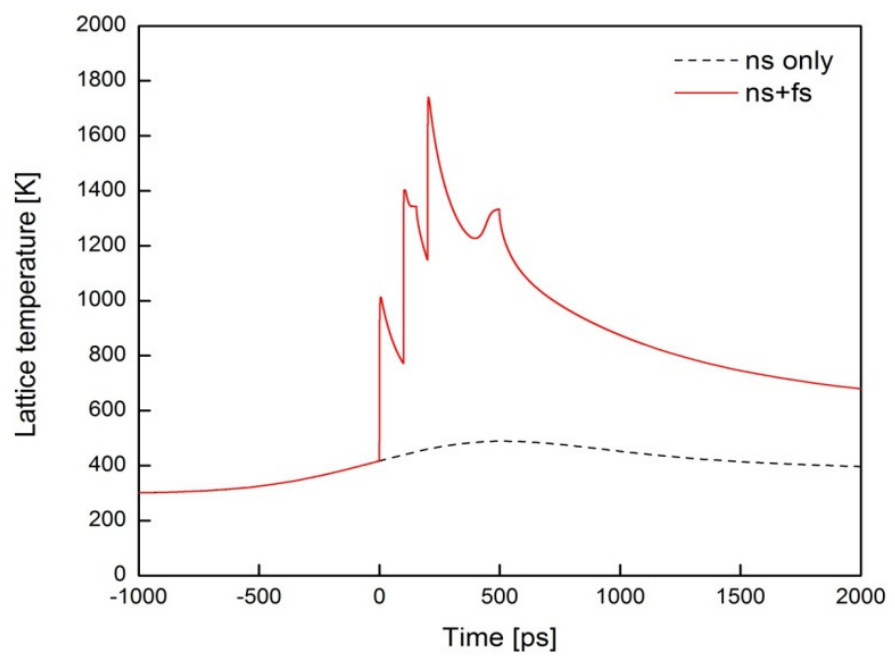

(c)

Fig. 6 Lattice temperatures at the irradiated surface induced by the dual laser beam of a nanosecond pulse and a three femtosecond pulses train: (a) $t_{\text {sep }}=1 \mathrm{ps}$, (b) $t_{\text {sep }}=10 \mathrm{ps}$, and (c) $t_{\text {sep }}=100 \mathrm{ps}$. 


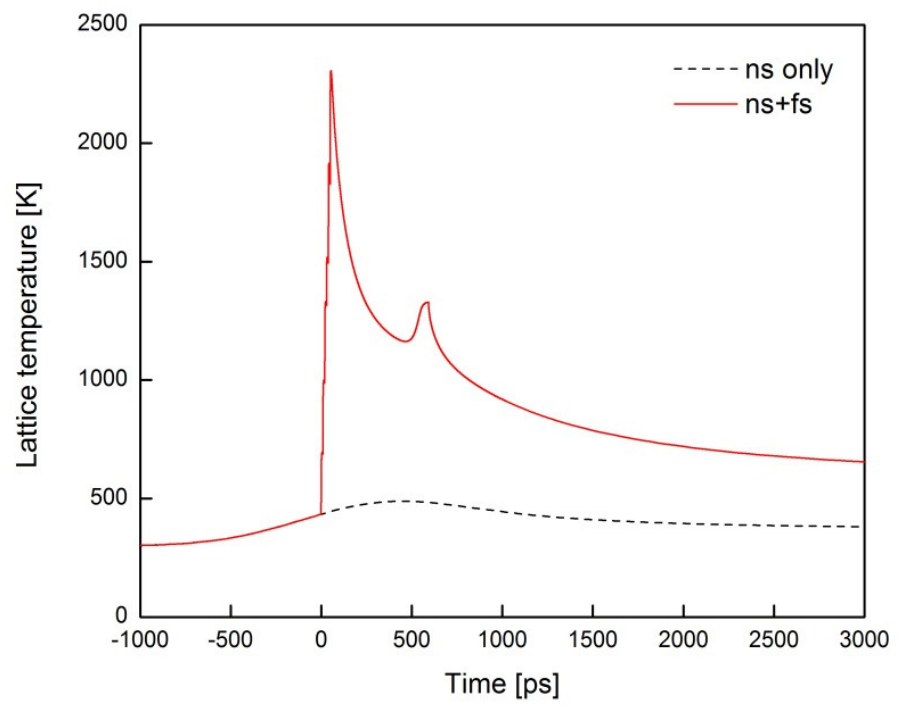

Fig. 7 Lattice temperatures at the irradiated surface induced by the dual laser beam of a nanosecond pulse and a six femtosecond pulses train with $t_{\text {sep }}=10 \mathrm{ps}$.

$2306 \mathrm{~K}$, which is lower than $2656 \mathrm{~K}$ produced by the dual beam with the 3-pulse train and the same separation time. Further simulations for the dual beam with a 6-pulse train give the maximum lattice temperature $2928 \mathrm{~K}$ for the 1-ps separation time and $1402 \mathrm{~K}$ for the 100 -ps separation time, compared to $2793 \mathrm{~K}$ and $1741 \mathrm{~K}$ for the dual beam with a 3-pulse train, respectively.

From the above results for the dual laser beams, it can be concluded that with the recent laser pulse train technology, the lattice temperature response can be enhanced by setting the pulse separation time as short as possible, for example $1 \mathrm{ps}$.

\section{CONCLUSIONS}

The temperature-dependent optical and thermophysical properties are incorporated into the semi-classical two-temperature model to study thermal response for a copper film irradiated by a femtosecond laser pulse train and by an integrated dual laser beam of a nanosecond pulse with a femtosecond pulse train. To study the effects of pulse number and separation time on the thermal response, two laser trains, consisting of three and six pulses each, and three separation times between pulses, $1 \mathrm{ps}, 10 \mathrm{ps}$ and $100 \mathrm{ps}$, are examined. In the numerical analysis, the total fluences are the same for all the trains, $1.0 \mathrm{~J} / \mathrm{cm}^{2}$. All the pulses in each train have the same fluence, wavelength $800 \mathrm{~nm}$, and duration 100 fs. The parameters of the nanosecond laser pulse are: $\lambda=800 \mathrm{~nm}, t_{p}=1$ $\mathrm{ns}$, and fluence $0.5 \mathrm{~J} / \mathrm{cm}^{2}$. For comparison, the results of the copper film irradiated by a single femtosecond pulse of fluence $1.0 \mathrm{~J} / \mathrm{cm}^{2}$ are also presented. The following conclusions are drawn:

(1) The change of optical properties at high temperatures is the main factor that alters the thermal response of a metal film induced by ultrafast laser heating.

(2) Pulse separation time controls the incubation effect. The shorter the separation time is, the less the heat is conducted. Thus, lattice temperature can be enhanced by a laser pulse train with a shorter pulse separation time, especially for separation time less than $10 \mathrm{ps}$.

(3) To obtain higher lattice temperature, more pulses should be used in a train with shorter pulse separation time such as $1 \mathrm{ps}$ and fewer pulses in a train with longer pulse separation time such as $100 \mathrm{ps}$.

(4) Although the peak power of a nanosecond laser pulse is very small compared to that of a femtosecond laser pulse at similar level of fluence, combining a fs-pulse train to a nanosecond laser pulse can enhance the thermal response, especially for shorter pulse separation time.

(5) The lattice temperature produced by a dual laser beam of a nanosecond pulse and a fs-pulse train can be increased by setting the pulse separation time as short as possible, for example 1 ps.

\section{NOMENCLATURE}

$A_{e} \quad$ material constants for electron relaxation time

$B \quad$ weighing factor of Lorentz oscillators

$C$ heat capacity $\left(\mathrm{J} / \mathrm{m}^{3}-\mathrm{K}\right)$

$c \quad$ speed of light in vacuum $(\mathrm{m} / \mathrm{s})$

$e \quad$ charge of an electron $(\mathrm{C})$

$f_{l} \quad$ normal refractive index

$f_{2} \quad$ extinction coefficient

$f_{\text {rep }} \quad$ repetition rate of pulse train

G

$\hbar$

$h_{m}$

$J$

$k$

$k_{B}$

$k_{F}$

$L$

$m$

$m_{\text {opt }}$

$q$

$R_{g}$

$R_{g}$

S

S

$t$

$t_{p}$

$t_{\text {sep }}$

$T$

$T_{F}$

$T_{m}$

$u$

$v$

$u_{0}$

$x$

\section{Gre}

$\alpha$

$\beta$

$\varepsilon$

$\varepsilon$

$\varepsilon_{1}$

$\varepsilon_{2}$
$\varepsilon_{F}$

$\gamma_{D}$

$\mu_{0}$

$\rho$

$\tau$

$\tau$

$v_{e-p h}$

$\omega$

$\omega_{p}$

$\phi$

$\Gamma$

$\Omega$

Subscripts

e electron

$e q$ thermal equilibrium state

$i$ initial condition

$l \quad$ lattice

$\ell \quad$ liquid

$s \quad$ solid

se solid-liquid interface 


\section{REFERENCES}

Anisimov, S. I., Kapeliovich, B. L., and Perel'man, T. L., 1974, "Electron Emission from Metal Surfaces Exposed to Ultra-Short Laser Pulses," Soviet Physics JETP, 39 (2), 375-377.

Anisimov, S. I., Rethfeld, B., 1997, "On the Theory of Ultrashort Laser Pulse Interaction with A Metal," Proceedings of SPIE, 3093, 192-203.

Baheti, K., 2010, An Axisymmetric Interfacial Tracking Model for Melting-Vaporization-Resolidification in a Thin Metal Film Irradiated By Pico To Femtosecond Pulse Lasers, Master's Thesis, University of Missouri, Columbia, MO.

Chen, J. K. and Beraun, J. E, 2001, "Numerical Study of Ultrashort Laser Pulse Interactions with Metal Films," Numerical Heat Transfer A, 40, $1-20$ http://dx.doi.org/10.1080/104077801300348842

Chen, J. K. and Beraun, J. E., 2005, "Investigation of Ultrafasr Laser Ablation Using a Semiclassical Two-Temperature Model," Journal of Directed Energy, 1, 261-274. http://dx.doi.org/10.1080/104077801300348842

Chen, J. K., Zhou, D. Y., and Beraun, J. E., 2006, "A Semiclassical Two Temperature Model For Ultrafast Laser Heating," International Journal of Heat and Mass Transfer, 49(1-2), 307-316. http://dx.doi.org/10.1016/j.ijheatmasstransfer.2005.06.022

Chowdhury, I. H., Xu, X., and Weiner, A. M., 2003, "Ultrafast Pulse Train Micromachining," Proceedings of SPIE, 4978, 138-146.

Du, G., Chen, F., Yang, Q., Si, J., and Hou, X., 2011, "Ultrafast Thermalization Characteristics in Au Film Irradiated by Temporally Shaped Femtosecond Laser Pulses," Optics Communications, 284, 640645.

http://dx.doi.org/10.1016/j.optcom.2010.09.061

Etchegoin, P. G., Le Ru, E. C., and Meyer, M., 2006, “An Analytical Model for the Optical Properties of Gold," Journal of Chemical Physics, 125, 164705.

http://dx.doi.org/10.1063/1.2360270

Faghri, A., and Zhang, Y., 2006, Transport Phenomena in Multiphase Systems, Elsevier Academic Press, Burlington, MA.

Fisher, D., Fraenkel, M., Henis, Z., Moshe, E., and Eliezer, S., 2001, "Interband and Intraband (Drude) Contributions to Femtosecond Laser Absorption in Aluminum," Physical Review E, 65, 016409. http://dx.doi.org/10.1103/PhysRevE.65.016409

Fox, M., 2010, Optical Properties of Solids, Oxford University Press, Oxford.
Huang, J., Zhang, Y., Chen, J. K., and Yang, M., 2011, "Modeling of Ultrafast Phase Change Processes in a Thin Metal Film Irradiated by Femtosecond Laser Pulse Trains," Journal of Heat Transfer, 133, 031003. http://dx.doi.org/10.1115/1.4002444

Jiang, L., and Tsai, H.-L., 2005, "Repeatable Nanostructures in Dielectrics by Femtosecond Laser Pulse Trains," Applied Physics Letters, 87, 151104. http://dx.doi.org/10.1063/1.2093935

Jiang, L., and Tsai, H.-L., 2007, "Modeling of Ultrashort Laser PulseTrain Processing of Metal Thin Films," International Journal of Heat and Mass Transfer, 50(17-18), 3461-3470. http://dx.doi.org/10.1016/j.ijheatmasstransfer.2007.01.049

Klemens, G., and Williams, R. K., 1986, "Thermal Conductivity of Metals and Alloys," International Metals Reviews, 31(5), 197-244.

Kuo, L.-S., and Qiu, T., 1996, "Microscale Energy Transfer during Picosecond Laser Melting of Metal Films," Proceedings of 1996 ASME National Heat Transfer Conference, Atlanta, GA.

Li, X., Jiang, L., and Tsai, H.-L., 2009, "Phase Change Mechanisms during Femtosecond Laser Pulse Train Ablation of Nickel Thin Films," Journal of Applied Physics, 106, 064906. http://dx.doi.org/10.1063/1.3223331

Ren, Y., Chen, J. K., and Zhang, Y., 2011, "Optical Properties and Thermal Response of Copper Films Induced by Ultra-Short Pulsed Lasers," Journal of Applied Physics, 110, 113102. http://dx.doi.org/10.1063/1.3662897

Ren, Y., Chen, J. K., and Zhang, Y., 2012, "Modeling of Ultrafast Phase Changes in Metal Films Induced by an Ultrashort Laser Pulse Using a Semi-classical Two-temperature Model," International Journal of Heat and Mass Transfer, 55(5-6), 1620-1627.

http://dx.doi.org/10.1016/j.ijheatmasstransfer.2011.11.018

Sim, H. S., Park, S., Kim, T.-H., Choi, Y. K., Lee, J. S., and Lee, S. H., 2010, "Femtosecond Laser Pulse Train Effect on Optical Characteristics and Nonequilibrium Heat Transfer in Thin Metal Films," Materials Transactions, 51(6), 1156-1162. http://dx.doi.org/10.2320/matertrans.M2010009

Stoian, R., Boyle, M., Thoss, A., Rosenfeld, A., Korn, G., and Hertel, I. V., 2002, "Laser Ablation of Dielectrics with Temporally Shaped Femtosecond Pulses," Applied Physics Letters, 80(3), 353-355. http://dx.doi.org/10.1063/1.1432747

Zhang, Y., and Chen, J. K., 2008, "An Interfacial Tracking Method for Ultrashort Pulse Laser Melting and Resolidification of a Thin Metal Film," Journal of Heat Transfer, 130, 062401. http://dx.doi.org/10.1115/1.2891159 